\title{
Selection of Spectral Filters for Optical Demultiplexer- Same Filter Different Source
}

\author{
Mohammad Syuhaimi Ab-Rahman, Hadiguna and Latifah Sarah Supian \\ Department of Electrical, Electronics and System Engineering, \\ Spectrum Technology Research Group (SPECTECH), \\ Faculty of Engineering and Built Environmental, \\ University Kebangsaan Malaysia, 43600 UKM Bangi, Selangor, Malaysia
}

Received 2012-07-09, Revised 2012-08-24; Accepted 2013-11-08

\begin{abstract}
This study aims to evaluate the effectiveness of selected color filter film for demultiplexer construction where only signals with certain wavelengths are allowed to pass through the demultiplexer while the other signal that carrying different wavelengths are absorbed. Characterization testing and analysis is done based on recorded data. Demultiplexer components are characterized by a Same Filter Different Sources (SFDS) and performance of transmitted signal has been measured. Demultiplexer developed is effective in short range communication, using low cost components and simple techniques. Communication range for short range communication is maximized. Only signals with certain wavelengths are allowed to pass through the multiplexer. The rest are absorbed. Several measures are made to ensure efficiency, such as testing and measurement on parametres contribting to loss. Best filters are selected based on their ability to block certain wavelengths while letting others ro pass through efficiently.
\end{abstract}

Keyword: Plastic Optical Fiber, Demultiplexer, Spectral, Filter, Output Power

\section{INTRODUCTION}

The field of 'green technology' encompasses a continuously evolving group of methods and materials, from techniques for generating energy to non-toxic cleaning products. The present expectation is that this field will bring innovation and changes in daily life of similar magnitude to the 'information technology' explosion over the last two decades. In these early stages, it is impossible to predict what 'green technology' may eventually encompass (Gupta and Khurana, 2010).

Today, the whole world of telecommunications and information communities is facing a more and more serious challenge, namely on one side the transmitted multimedia-rich data are exploding at an astonishing speed and on the other side the total energy consumption by the communication and networking devices and the relevant global $\mathrm{CO} 2$ emission are increasing terribly. It has been pointed out that currently $3 \%$ of the world-wide energy is consumed by the Information and Communications Technology (ICT) infrastructure that causes about $2 \%$ of the world-wide $\mathrm{CO} 2$ emissions, which is comparable to the worldwide $\mathrm{CO} 2$ emissions by airplanes or one quarter of the world-wide $\mathrm{CO} 2$ emissions by cars (Janota and Hrbcek, 2011) Nowadays, optical fiber become an alternative transmission media replacing copper cable for short-haul communication and has been utilized by advanced country in Asia such as South Korea, Hong Kong and Japan. Malaysian government's efforts to bring Malaysia into the era of 'new economy' have created demand in multimedia and communications networks for a better efficiency, especially for broadband services. It can be seen from broadband penetration rate are closely to 0.55 per thousand of population in 2005 , as significantly increased from previous year in 2001 namely by 1.5 per thousand of population.

\footnotetext{
Corresponding Author: Mohammad Syuhaimi Ab-Rahman, Department of Electrical, Electronics and System Engineering, Spectrum Technology Research Group (SPECTECH), Faculty of Engineering and Built Environmental, University Kebangsaan Malaysia, 43600 UKM Bangi, Selangor, Malaysia
} 


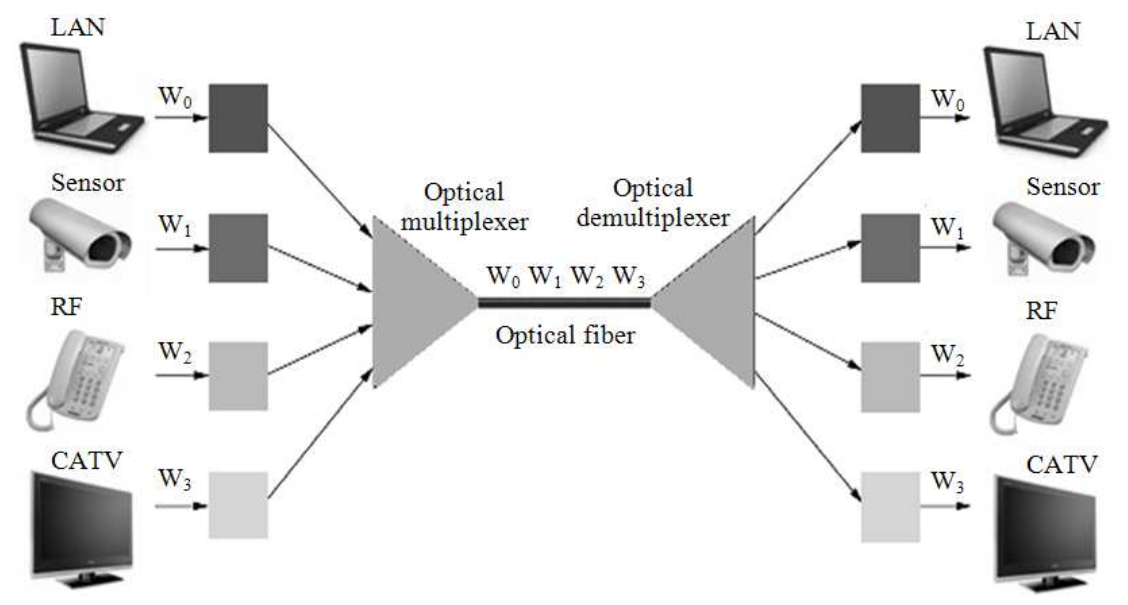

Fig. 1. Wavelength Division Multiplex (WDM) with an optical multiplexer and demultiplexer

Recently, silica or Glass Fiber (GOF) has become a 'trendsetter' in Fiber to the Home (FTTH) technology to provide a high capacity communication data to be transmitted to a user from central office. But, not much people or even service provider alerts from the safety concern of the technology, especially for user. Laser, as the best transmission media to be utilized together with GOF could be very dangerous once the leakage occurred from the body structure of the GOF. A very high intensity ray of light released by fiber can possibly burn a human retinas and lead to a permanent blind.

Combination between Plastic Optical Fiber (POF) which very suitable to a Light Emitting Diode (LED) technology can be seen as the best solution to provide a communication data services which is safer and not mentioning about the lowest price we can get for the capital and fabrication cost. POF links are becoming increasingly popular for applications such as computer or peripheral connections, control and monitoring, board interconnects and even domestic hi-fi systems. Unlike GOF, POF remains flexible while having a large diameter core and high numerical aperture (Kuzyk, 2010), lead to high capacity they can bring along the fiber.

Moreover, the fiber is easy to handle with the potential for constructing networks using simple conductor and easy installation procedures while retaining some of the advantages of optical fiber such as Electromagnetic Interference (EMI) immunity, nonconducting cable, small size and security. Another feature is the use of visible light to transmit information (Grzemba, 2008).

Recent communication system over POF desires increasingly more bandwidth and therefore the Wavelength Division Multiplexing (WDM) system is the solution that allows the transmission of information over more than just a single wavelength (color) and thus greatly increases the POF's bandwidth. WDM is a technique that multiple signals are carried together as separate wavelengths (color) of light in a multiplexed signal. As shown is Fig. 1, Wavelength Division Multiplexer is the first passive device required in WDM-POF system and it functions to combines optical signals from multiple different singlewavelength end devices onto a single fiber.

Ab-Rahman et al. (2011b) has demonstrated for the first time the POF devices can be fabricated by the skillful hand. The temperature, stress and splitting technique are the most important parameters to fabricate low loss device. With some modification the device can be used for the extended function such as demultiplexer which is fabricated from uniformity optical splitter (AbRahman et al., 2011a). In WDM-POF system, many transmitters with different lights color to carry single information. For example, red light with $650 \mathrm{~nm}$ wavelength modulated with Ethernet signal while blue, green and yellow lights carry image information, Radio Frequency (RF) and television signal, respectively (AbRahman et al., 2012c). Wavelength Division Multiplexer is the first passive device required in WDM-POF system and it functions to combines optical signals from multiple different single-wavelength end devices onto a single fiber (Ab-Rahman et al., 2012b). Conceptually, the same device can also perform the reverse process with the same WDM techniques, in which the data stream with multiple wavelengths decomposed into multiple single wavelength data streams. The reverse process is called as demultiplexing. Conceptually, POF splitter has similar function, operates to couple or combine several optical data pulse as a single coupled signal. 


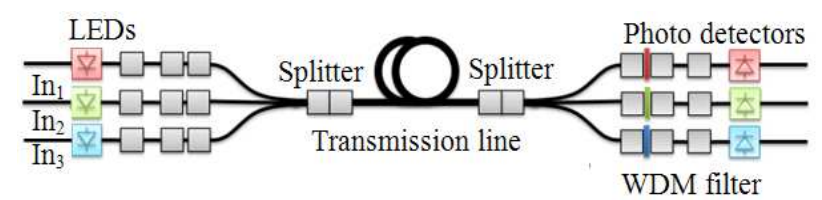

Fig. 2. Eco-friendly-WDM-POF network architecture using $1 \times 3$ splitter and color filters the splitter can also be used as multiplexer and the splitter-filter combination work as a demultiplexer

Hence, the development of wavelength division multiplexer based on POF splitter is possible. A low-cost solution for POF-WDM system application will be presented.

A novel fused POF splitter will fabricated by a fusion technique as reported by Ab-Rahman et al. (2011b), as an effective transmission media to split and recombine a number of different wavelengths which represents different signals. Three different wavelengths will be fully utilized to transmit three different sources of systems; LAN connection Network, radio (audio) and video transmission system. Red LED which in $665 \mathrm{~nm}$ wavelength capable to download and upload data through Ethernet cable while green LED in 520nm wavelength can transmit a video image generated from DVD player or CCTV system and blue LED with 470nm wavelength represents an audio transmission system inside the house (Ab-Rahman et al., 2012a). Special filter will be placed between the splitter and receiver-end to make sure the entire WDM system can select a single signal as desired. For the filter design which able to eliminate unwanted signal and select the wavelength of the system as desired as shown in Fig. 2. Some parameters, such as optical output power, power losses, optical noise to ratio and crosstalk of the devices can be observed and not mentioning about the effect of filter placement and the efficiency of the WDM-POF system itself.

\subsection{Objectives}

The objectives of characterization and analysis testing are:

- To develop demultiplexer component and optical splitter that provide optimal results when applied to the data transmission systems

- To evaluate the effectiveness of selected color filter film for demultiplexer construction where only signals with certain wavelengths are allowed to pass through the demultiplexer while the other signals carrying different wavelengths are absorbed

- To find maximum distance of developed components where transmitted signal can be delivered quickly and effectively
- To ensure the developed components can deliver the best performance in short range communication system without lot of costs

Characterization testing and analysis are done based on recorded data. The developed demultiplexer components are categorized a Same Filter Different Sources (SFDS) and a performance of transmitted signal by the presence of interference signal (noise and cross talk) are measured.

For final analysis, the outcomes are:

- The developed demultiplexer is effective to be applied in short range communication system by using low-cost components and implementation techniques that are not too complicated

- Maximum distance can be achieved for short range communications system by using the components developed

- Signals that carry different wavelengths are absorbed or prevented from being transmit through the developed demultiplexer with filter film. Only signals with certain wavelengths are allowed to pass through the demultiplexer

- Signal transmitted through optical fiber and optical splitter by using LED light source is tested for the maximum distance that can be achieved for the purpose of data transmission. Parameters that have contributed to the loss of signals (which are carried by the components developed) are tested and measured. It is done to ensure the interference that exists in data transmission system does not affect the total emitted signal or reduced the performance of system that was developed in order to maintain the performance of signal transmission efficiency

\subsection{Same Filter Different Source (SFDS)}

For the characterization of same sources and different filter film, a light source of red, green and blue LED are used as raw materials. Based on Fig. 3, the analysis for sample 6 red filters (\#332) showed the least insertion loss and also a small reduction in output power. It shows the efficiency of filter film that is used. While sample 8 (\#4690) is a sample film of very thick color in which only small portion of wavelengths can pass through the filter film. The consumption of epoxy-resin adhesive also contributed to high insertion loss for this filter film.

Experiments are also performed by injecting blue LED light source (Fig. 4) through optical fiber and pass through the filter film that has been provided. Data analysis shows that sample 6 gives the best reading of highest power reduction compared to other samples. 
Mohammad Syuhaimi Ab-Rahman et al. / Journal of Computer Science 9 (4): 413-420, 2013

The reduction in output power (UW) of red filter samples that injected to red LED

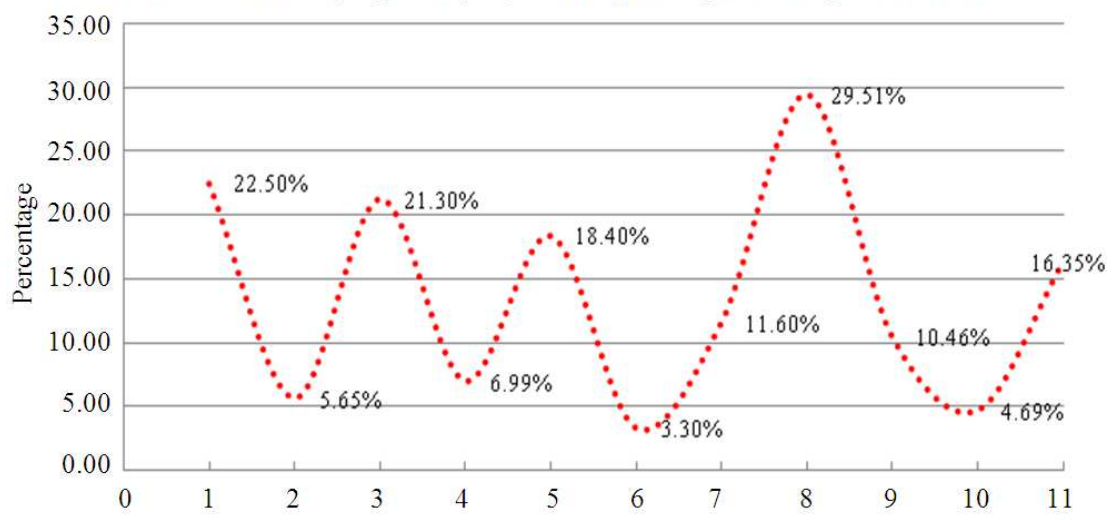

Fig. 3. The reduction in output power $(\mu \mathrm{W})$ of red filter samples that has injected to red LED

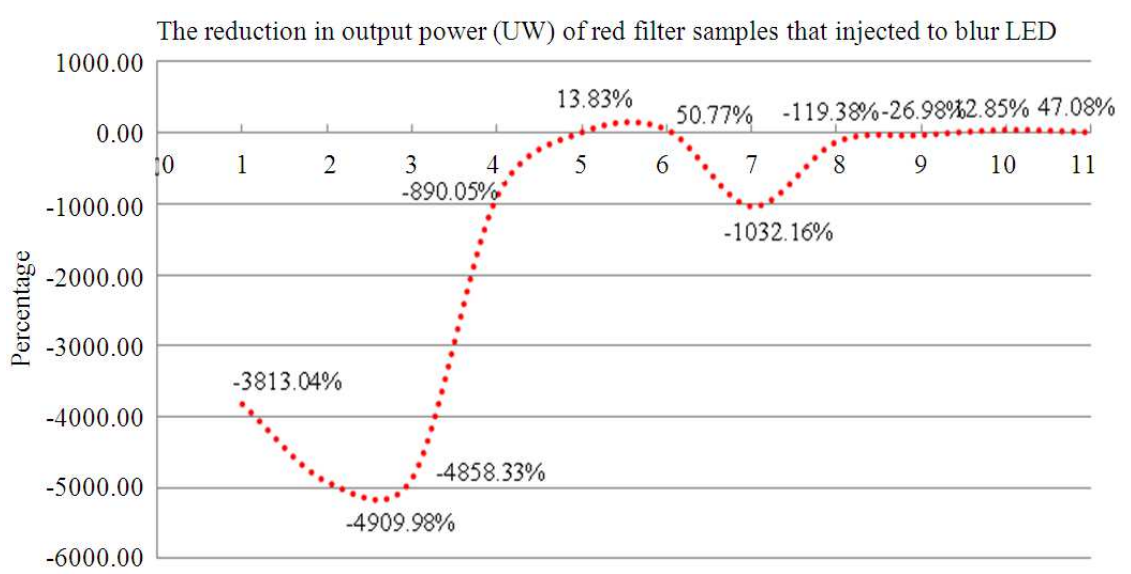

Fig. 4. The reduction in output power $(\mu \mathrm{W})$ of red filter samples that has injected to blue LED

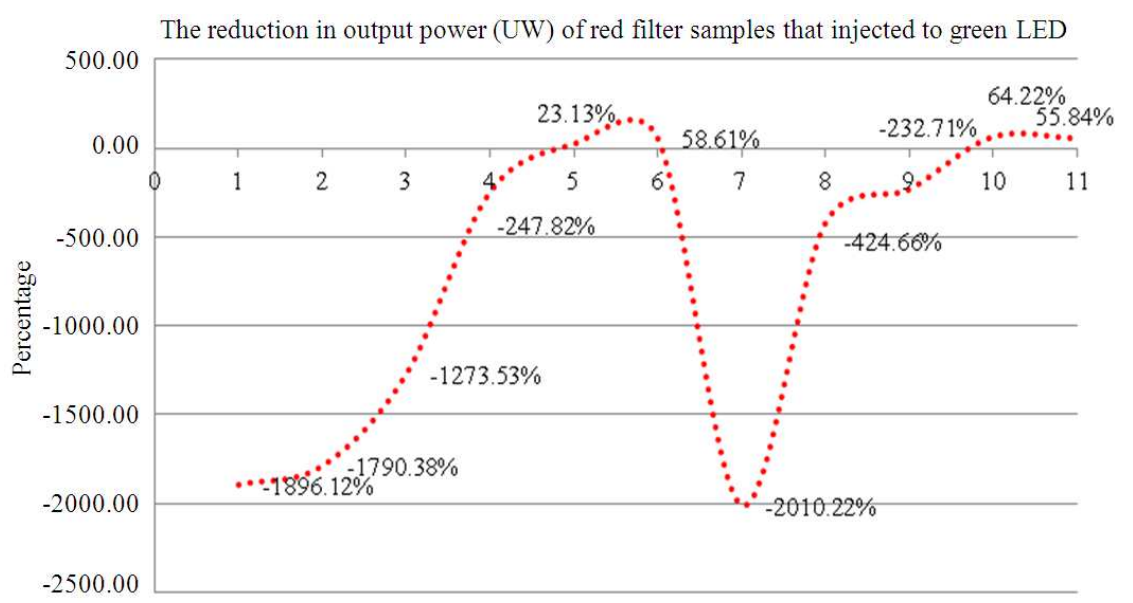

Fig. 5. The reduction in output power $(\mu \mathrm{W})$ of red filter samples that has injected to green LED 


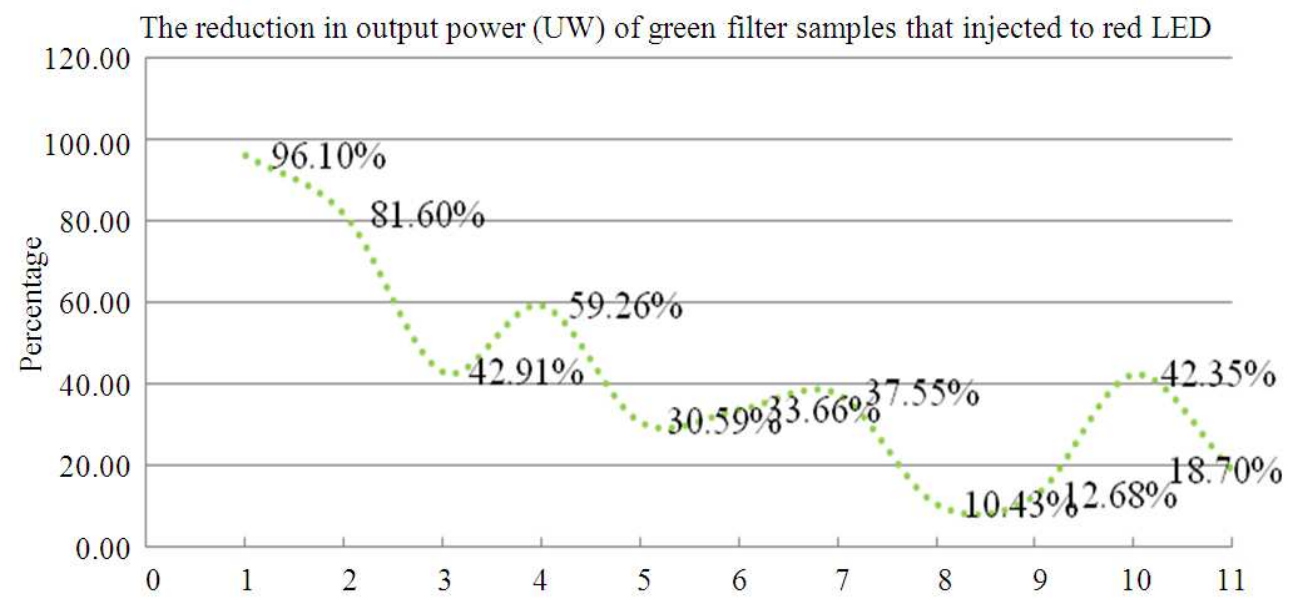

Fig. 6. The reduction in output power $(\mu \mathrm{W})$ of green filter samples that has injected to red LED

Sample 6 prevent the red LED wavelength to pass through, thus inhibiting unwanted data from being sent to the final receiver. Negative percentage values indicate the increasing output power, in which wavelength of LED light sources are allowed to enter through the filter film sample. Therefore, the other sample is not selected as a filter film in order to be used in construction of demultiplexer.

Further testing on red filter samples is done by injecting the green LED (Fig. 5) light source through fiber optics and pass through the filter film sample that has been provided. Data analysis from plotted graph below shows a sample of 10 and 6 that give a good reading with high reduction in power compared to other samples. Sample 10 and 6 prevents red LED wavelength to pass through, thus inhibiting unwanted data from being sent to the final receiver. However, based on previous characterization analysis by injecting red and blue LED, sample 6 provides optimal results and this samples can be used in demultiplexer process.

For blue and green filter film that has been injected with red LED, theoretically, both of that filters film will absorb or block red wavelengths from pass through the film. From data analysis of green sample that has been injected with red LED (Fig. 6), sample 1 (\#89) prevent most of the wavelength signal that pass through the filter film, thus the efficiency has decreased by $98 \%$ based on reduction in Output Power (UW). Sample 2 also shows a high output power loss followed by sample of 4 , 3,10 and 7 .

This film also absorbs most of the emitted red LED wavelength. Insertion loss rate recorded a lot of decreases and the efficiency rate also has been decrease due to the severe decline in output power.

Based on the analytical characterization of green filter sample that has been injected with blue light source (Fig. 7), a sample of 2 filter film (\#86) has the highest loss in output power followed by a sample of $1,10,3,4$, 6 and 7. It shows that the filter film is able to prevent different light sources with different wavelengths from entering through the green filter film further characterization test is performed by injecting green filter film sample with green LED light source (Fig. 8). Based on analysis from the plotted graph, sample 1 does not give a notable performance where the filter film sample prevent $40 \%$ of green light source emissions to pass through. Also, the output power loss is higher compared to samples 2, 4, 6 and 7. Based on analytical characterization that was done by injecting red and blue LED light source, sample 2 (\#86) and sample 7 (\#3304) gave the best reading compared to other filter film sample. Both of these samples are used in the construction of demultiplexer in this experiment. Negative percentage values show that the increasing output power, in which wavelength of LED light sources are allowed to enter through the filter film sample. Therefore, the other sample is not selected as a filter film in order to be used in construction of demultiplexer.

Further testing on blue filter samples is done by injecting the red, blue and green LED light source through fiber optics and blue filter film. The readings of insertion loss and output power are taken using the power meter. Analytical characterization is performed by evaluating the rate of decline in output power for each blue sample. 
Mohammad Syuhaimi Ab-Rahman et al. / Journal of Computer Science 9 (4): 413-420, 2013

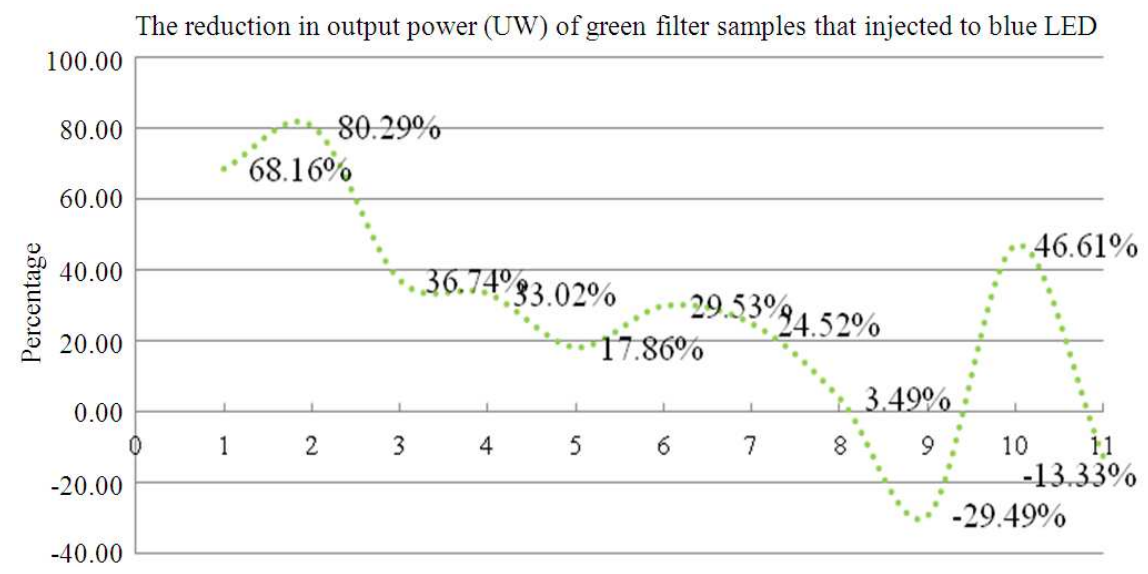

Fig. 7. The reduction in output power $(\mu \mathrm{W})$ of green filter samples that has injected to blue LED

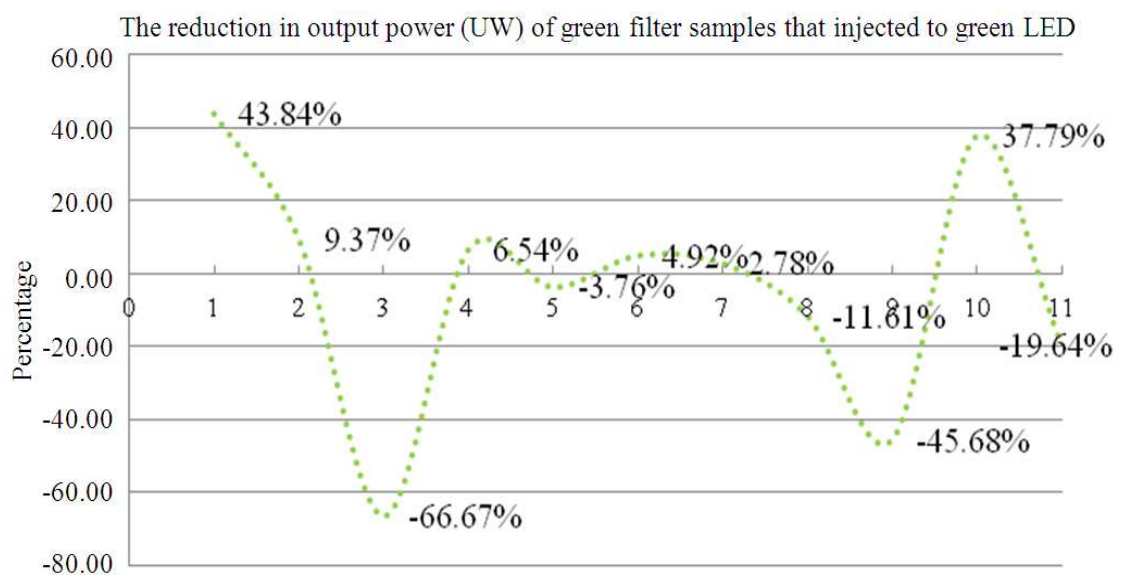

Fig. 8. The reduction in output power $(\mu \mathrm{W})$ of green filter samples that has injected to green LED

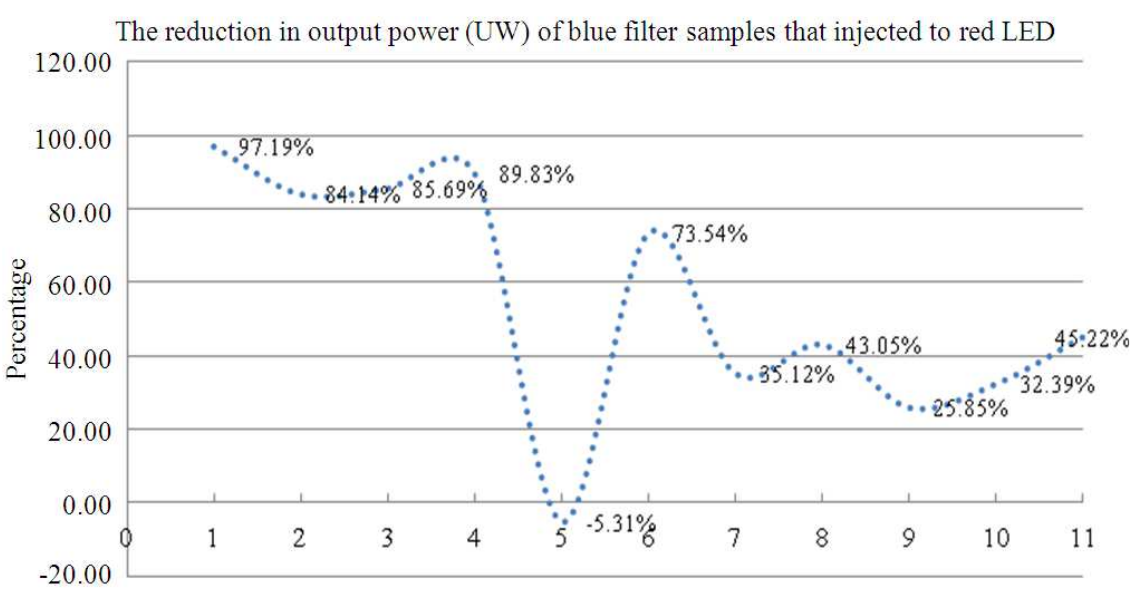

Fig. 9. The reduction in output power $(\mu \mathrm{W})$ of blue filter samples that has injected to red LED 
Mohammad Syuhaimi Ab-Rahman et al. / Journal of Computer Science 9 (4): 413-420, 2013

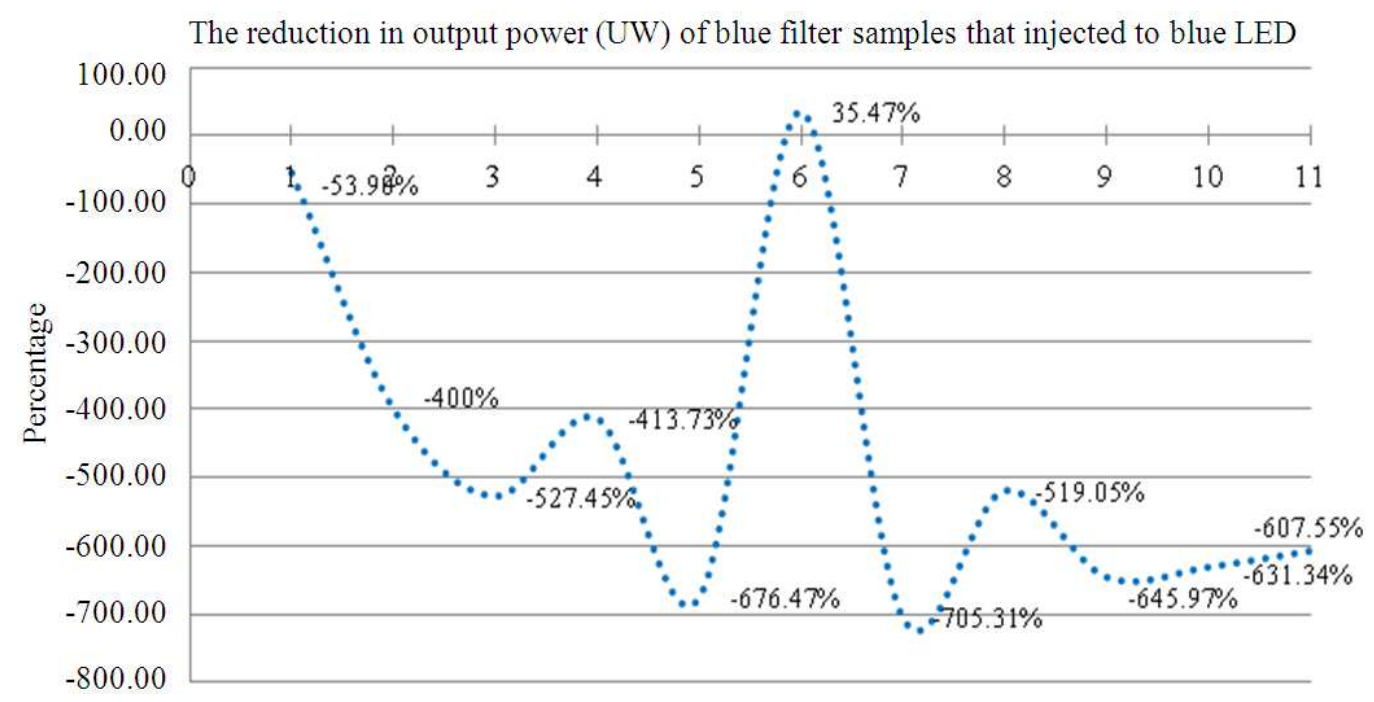

Fig. 10. The reduction in output power $(\mu \mathrm{W})$ of blue filter samples that has injected to blue LED

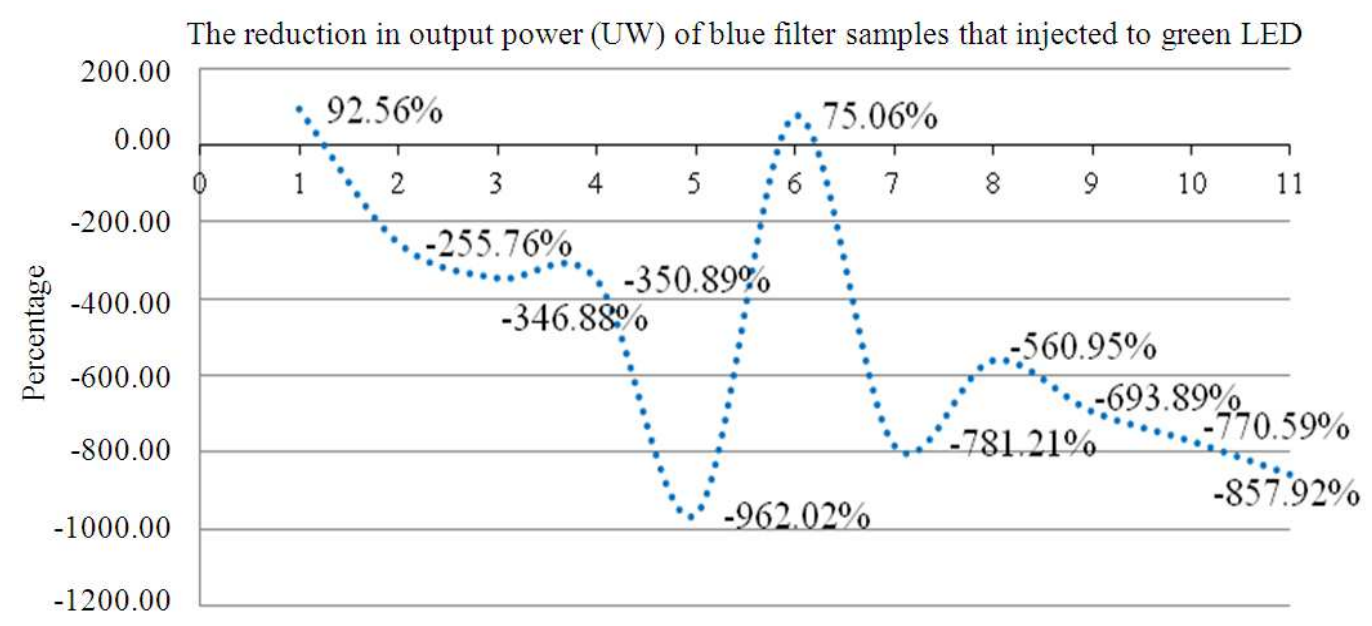

Fig. 11. The reduction in output power $(\mu \mathrm{W})$ of blue filter samples that has injected to green LED

Based on the plotted graph as depicted in Fig. 9, sample 1 of blue filter film that has been injected with red LED recorded the highest loss in output power followed by a sample of 4, 3, 2, 6 and 11. Readings on negative values are not chosen because the film allows light source with different wavelengths to pass through it.

Figure 10 shows the output power loss when blue filter sample was injected with blue LED light source. Based on plotted graphs, sample 6 shows the minimum reduction in output power where almost $65 \%$ of blue light emission are allowed to enter through it. Analysis is also performed on blue filter film sample where the green LED source light is emitted (Fig. 11).

\section{CONCLUSION}

Only sample 1 and 6 of blue filter film are absorbed or block most of the emitted green LED signal wavelength. The other samples gave negative readings because they allow the green LED light source with different wavelengths to pass through. Based on analytical testing characterization that was done for blue filter film samples, only sample 6 (\#62) gives the best reading compared to other samples. Sample 6 of filter film shows the optimal efficiency, where this filter film allows $65 \%$ of blue LED light to pass through and sending data to the end receiver. This filter films also 
prevent or absorb $70 \%$ of other LED light wavelengths to pass through. Therefore, this sample is used in the construction of demultiplexer during this experiment.

\section{REFERENCES}

Ab-Rahman, M.S., L.S. Supian, H. Guna, M.H. Harun and K. Jumari, 2012b. Performance of thin-film as wavelength filter in wdm-pof network. Am. J. Applied Sci., 9: 180-185.

Ab-Rahman, M.S., L.S. Supian, H. Guna, M.H. Harun and K. Jumari, 2012c. Low-loss hand-made demultiplexer using transparent color filter in WDM-POF network for short-haul communication system. J. Comput. Sci., 8: 494-498.

Ab-Rahman, M.S., M.H. Harun and K. Jumari, 2011a. The importance of uniformity to demultiplexer device fabrication for pof based small world communication. Advances Natural Applied Sci., 5: 166-170.

Ab-Rahman, M.S., M.H. Harun and K. Jumari, 2011 b. Second generation of low-cost fused tapered (LFT) splitters based on POF technology. J. Sci. Industrial Res., 70: 435-442.
Ab-Rahman, M.S., N.N.S. Mohammad, M.H. Harun and L.S. Sufian 2012a. Customer-made $3 \times 3$ polymer optical fiber splitter based on green technology using fusion technique. J. Comput. Sci., 8: 591-598. DOI: 10.3844/jcssp.2012.591.598

Grzemba, A., 2008. Most: The Automotive Multimedia Network. 1st Edn., Franzis, Poing, ISBN-10: 9783772353161, pp: 383.

Gupta, P. and H. Khurana, 2010. Public entrepreneurship: A dynamic strength for budding green technology. Proceedings of the 4th National Conference, Feb. 25-26, Computing For Nation Development New Delhi, India.

Janota, A. and J. Hrbcek, 2011. Slovak ETC system implemented-what next? Trans. Syst. Telematics, 104: 30-37. DOI: 10.1007/978-3-642-16472-9_3

Kuzyk, M.G., 2010. Polymer Fiber Optics: Materials, Physics and Applications. 1st Edn., Taylor and Francis, ISBN-10: 1420017802, Boca Raton, pp: 399. 